Abstract

\title{
Novel Fabrication Technique of Superimposed LPG with Different Grating Pitches for the Simultaneous Detection of Refractive Index and Temperature ${ }^{+}$
}

\author{
Cosimo Trono ${ }^{1, *}$, Federico Valeri ${ }^{1,2}$ and Francesco Baldini ${ }^{1}$ \\ 1 Istituto di Fisica Applicata "Nello Carrara", Consiglio Nazionale delle Ricerche, \\ 50019 Sesto Fiorentino, Italy \\ 2 Dipartimento di Fisica, Università degli Studi di Firenze, 50019 Sesto Fiorentino, Italy \\ * Correspondence: c.trono@ifac.cnr.it \\ + Presented at the 7th International Symposium on Sensor Science, Napoli, Italy, 9-11 May 2019. \\ Published: 31 July 2019
}

Optical fiber sensors based on in-fiber Long Period Gratings (LPG) have attracted considerable interest in the context of environmental, chemical and biochemical sensing. LPG is characterized by a grating period in the range of hundreds of micrometer giving rise to the coupling between the fundamental core mode and a discrete set of forward propagating cladding modes. The specific coupling between the modes generates in the transmission spectrum a series of attenuation bands dependent on the grating pitch. In the present work is described a technique for the inscription of superimposed LPGs with different grating pitches, which allows the coupling to two different cladding modes whit resonant wavelengths within a relatively small wavelength band. These cladding modes will exploit different sensitivities to RI and temperature making it possible the simultaneous detection of these two parameters. LPGs are usually fabricated by using an excimer UV laser with emission at $248 \mathrm{~nm}$, shaped by means of a slit, and the point by point technique, that allows to obtain a square-wave core RI modulation, although the sinusoidal modulation should theoretically give rise to better results. The writing technique here proposed is based on the writing of an almostsinusoidal modulation of the core refractive index by sampling the sinusoidal function with small rectangular modulations obtained by a very small aperture slit $(\sim 30 \mu \mathrm{m})$. Although this technique does not give rise to significant advantages in the writing of a single LPG, is here demonstrated to be essential for the inscription of superimposed LPGs.

Acknowledgements: Regione toscana, programma operativo regionale fesr 2014-2020, bando n.2: progetti strategici di ricerca e sviluppo delle mpmi, progetto cirebi, Componenti Intelligenti con REticoli di Bragg Integrati.

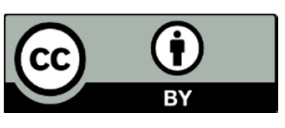

(C) 2019 by the authors. Licensee MDPI, Basel, Switzerland. This article is an open access article distributed under the terms and conditions of the Creative Commons Attribution (CC BY) license (http://creativecommons.org/licenses/by/4.0/). 